\title{
THE BAIRE CLASSIFICATION OF ORDINARY AND APPROXIMATE PARTIAL DERIVATIVES
}

\author{
LARRY E. SNYDER ${ }^{1}$
}

Introduction. It is well known that a function which is a derivative is in the first Baire class, and it is also known that a function which is an approximate derivative is in the first Baire class. The analogous statement for partial derivatives of functions of more than one variable is of course not true. For if $g$ is a nonmeasurable function of one variable, then the function $f(x, y)=x \cdot g(y)$ has a partial derivative which is nonmeasurable. Thus it is seen that a function of $n$ variables need be restricted somewhat in order for its partial derivative to be in the first Baire class. G. P. Tolstov [4] has shown that if $f$ is linearly continuous on a domain $G \subset R^{2}$ and if $\partial f / \partial x$ exists everywhere in $G$, then $\partial f / \partial x$ is in the first Baire class. In addition, he has given an example of a linearly continuous function of three variables whose partial derivative exists and is not in the first Baire class. In this paper we show that Tolstov's theorem remains true if approximate partial differentiation is used in place of ordinary partial differentiation.

Notation. Let $G$ denote a domain in $R^{2}$ and let $R^{+}$denote the positive real numbers. For a real-valued function $f$, let $\omega(f, p)$ denote the oscillation of $f$ at the point $p$.

Definitions. A function $f$ of $n$ real variables $x_{1}, x_{2}, \cdots, x_{n}$ is said to be linearly continuous if $f$ is continuous with respect to $x_{i}$ for each $i=1, \cdots, n$ whenever the other variables are held fixed.

Let $\left(x_{0}, y_{0}\right)$ be a fixed point in $G$. The set $S\left(x_{0}, y_{0}\right)=\left\{\left(x, y_{0}, r\right)\right.$ $\left.\in G \times R^{+}:\left|x-x_{0}\right| \leqq r\right\}$ is a vertical Stolz angle in the plane $y=y_{0}$ with vertex at $\left(x_{0}, y_{0}, 0\right)$.

Let $I_{0}$ be an open interval of the real line and let $f: I_{0} \rightarrow R$. The number $A$ is called the approximate derivative of the function $f$ at $x_{0}$ if there exists a set $E$, having $x_{0}$ as a density point, such that for $x \in E$ and $x \rightarrow x_{0}$ we have $\lim \left(\left(f(x)-f\left(x_{0}\right)\right) /\left(x-x_{0}\right)\right)=A$. The subscript "ap" will be used in connection with the usual notations for derivatives to indicate approximate derivatives.

Lemma 1. Let $\phi: G \times R^{+} \rightarrow R$, and suppose that $\left(x_{0}, y, r_{0}\right) \rightarrow \phi\left(x_{0}, y, r_{0}\right)$ is a continuous function of $y$ for each fixed $x_{0}$ and $r_{0}$, and suppose that for each point $\left(x_{0}, y_{0}\right)$ in $G$, the limit of $\phi(x, y, r)$ relative to $S\left(x_{0}, y_{0}\right)$

Received by the editors May 28, 1965.

${ }^{1}$ Supported in part by NSF Grant No. 18920. 
exists as $(x, y, r) \rightarrow\left(x_{0}, y_{0}, 0\right)$. If at each point $\left(x_{0}, y_{0}\right)$ in $G, f\left(x_{0}, y_{0}\right)$ is defined to be equal to this limit of $\phi(x, y, r)$, then the function $f: G \rightarrow R$ is in the first Baire class.

Proof. For the proof we use Baire's Theorem [2], that is, we verify that for any nonempty perfect set $P \subset G, f \mid P$ has a point of continuity. Suppose there is a nonempty perfect set $P \subset G$ such that $f \mid P$ has no point of continuity. For each positive integer $m$, let

$$
D_{m}=\left\{(x, y) \in P: \omega(f \mid P, \bar{x}) \geqq \frac{1}{m} \text { for } \bar{x}=(x, y)\right\} .
$$

Since $f \mid P$ is assumed to be totally discontinuous, $P=\cup_{m=1}^{\infty} D_{m}$. Then since $P$ is of the second category in itself, there is an integer $m_{0}$ and an open disc $\sigma$ in $G$ such that $D_{m_{0}}$ is dense in $P \cap \sigma$ and $P \cap \sigma \neq \varnothing$. Moreover, each $D_{m}$ is a closed set so that in fact $D_{m_{0}}$ contains $P \cap \sigma$. Let $Q=$ closure $(P \cap \sigma)$.

For each $m=1,2, \cdots$, let

$$
S(x, y, m)=\{(u, y, r) \in S(x, y): r \leqq 1 / m\}
$$

and let

$$
A_{m}=\left\{(x, y) \in Q: \xi \in S(x, y, m) \Rightarrow|\phi(\xi)-f(x, y)| \leqq \frac{1}{M}\right\},
$$

where $M=24 m_{0}$. It follows that $Q=\mathrm{U}_{m=1}^{\infty} A_{m}$, and since $Q$ is of the second category in itself, there is an integer $k_{0}$ and an open disc $\sigma^{\prime}$ such that $Q \cap \sigma^{\prime} \neq \varnothing$ and $A_{k_{0}}$ is dense in $Q \cap \sigma^{\prime}$.

Let $\left(x_{0}, y_{0}\right) \in P \cap \sigma \cap \sigma^{\prime}$. There is a smallest integer $m=m\left(x_{0}, y_{0}\right)$ for which $\left(x_{0}, y_{0}\right)$ is in $A_{m}$. Let $j=\max \left\{k_{0}, m\left(x_{0}, y_{0}\right)\right\}$. Since $\phi$ is continuous as a function of $y$ whenever $x$ and $r$ are fixed, there is a positive number $\delta$ such that $y_{0}-\delta<y<y_{0}+\delta$ implies

$$
\left|\phi\left(x_{0}, y, 1 / j\right)-\phi\left(x_{0}, y_{0}, 1 / j\right)\right|<\frac{1}{M} .
$$

Let $\tau$ denote the open disc in $G$ with $\left(x_{0}, y_{0}\right)$ as center and with radius equal to the smaller of $\delta$ and $1 / j$, and suppose $(u, v)$ is any point in $A_{k_{0}} \cap \tau$. Then $\left|v-y_{0}\right|<\delta$ and $\left|u-x_{0}\right|<1 / j$, so that

(1) the point $\left(x_{0}, v, 1 / j\right)$ is in $S(u, v, j)$ and

(2) $\left|\phi\left(x_{0}, v, 1 / j\right)-\phi\left(x_{0}, y_{0}, 1 / j\right)\right|<1 / M$.

Let $\left\{\left(x_{n}, y_{n}\right)\right\}$ be any sequence of points in $Q$ with $\left(x_{0}, y_{0}\right)$ as limit. There is an integer $N$ such that $i>N$ implies $\left(x_{i}, y_{i}\right)$ is in $V$, where $V=\tau \cap \sigma \cap \sigma^{\prime}$. Let $i$ greater than $N$ be fixed and let $q$ be the greater 
of $k_{0}$ and $m\left(x_{i}, y_{i}\right)$, where $m\left(x_{i}, y_{i}\right)$ is the smallest positive integer $m$ such that $\left(x_{i}, y_{i}\right)$ is in $A_{m}$. Then there is a positive number $\delta^{\prime}$ such that $y_{i}-\delta^{\prime}<y<y_{i}+\delta^{\prime}$ implies

$$
\left|\phi\left(x_{i}, y_{i}, 1 / q\right)-\phi\left(x_{i}, y, 1 / q\right)\right|<\frac{1}{M} .
$$

Since $A_{k_{0}}$ is dense in $V \cap Q$, there is a point $(u, v)$ in $A_{k_{0}} \cap V$ such that the distance from $(u, v)$ to $\left(x_{i}, y_{i}\right)$ is less than $\min \left\{\delta^{\prime}, 1 / q\right\}$. Then $\left|v-y_{i}\right|<\delta^{\prime}$ and $\left|u-x_{i}\right|<1 / q$, so that $\left(x_{i}, v, 1 / q\right)$ is in $S(u, v, q)$ and

$$
\left|\phi\left(x_{i}, v, 1 / q\right)-\phi\left(x_{i}, y_{i}, 1 / q\right)\right|<\frac{1}{M} .
$$

Since $(u, v)$ is in $A_{k_{0}} \cap \tau,(1)$ and (2) hold for $(u, v)$, and hence

$$
\begin{aligned}
\left|f\left(x_{0}, y_{0}\right)-f\left(x_{i}, y_{i}\right)\right| \leqq & \left|f\left(x_{0}, y_{0}\right)-\phi\left(x_{0}, y_{0}, 1 / j\right)\right| \\
& +\left|\phi\left(x_{0}, y_{0}, 1 / j\right)-\phi\left(x_{0}, v, 1 / j\right)\right| \\
& +\left|\phi\left(x_{0}, v, 1 / j\right)-f(u, v)\right| \\
& +\left|f(u, v)-\phi\left(x_{i}, v, 1 / q\right)\right| \\
& +\left|\phi\left(x_{i}, v, 1 / q\right)-\phi\left(x_{i}, y_{i}, 1 / q\right)\right| \\
& +\left|\phi\left(x_{i}, y_{i}, 1 / q\right)-f\left(x_{i}, y_{i}\right)\right|<\frac{6}{M}=\frac{1}{4 m_{0}} .
\end{aligned}
$$

However, this implies that $\omega\left(f \mid P,\left(x_{0}, y_{0}\right)\right) \leqq 1 / 2 m_{0}$, which is a contradiction.

It is not difficult to extend Lemma 1 to the $n$-dimensional case. For the statement of the lemma for the $n$-dimensional case, " $y$ " and " $y_{0}$ " are replaced by $(n-1)$-tuples of real numbers.

The next lemma concerns a convergent interval function defined by Goffman and Neugebauer in [1]. An extension of this notion will be used in proving the main theorem.

Lemma 2. Suppose that $g$ is an approximately derivable function defined on an open interval $J \subset R$. For $I \subset J$, let

$$
A(I ; k)=\left\{(u, v) \in I \times I: \frac{g(u)-g(v)}{u-v}>k\right\}
$$

and

$$
B(I ; k)=\left\{(u, v) \in I \times I: \frac{g(u)-g(v)}{u-v}<k\right\} .
$$


Then

$$
\sup \left\{k: \frac{|A(I ; k)|}{|I|^{2}}>\frac{1}{2}\right\}=\inf \left\{k: \frac{|B(I ; k)|}{|I|^{2}}>\frac{1}{2}\right\}=\phi(I) .
$$

Proof. For brevity let $A$ denote the set of $k$ 's for which $|A(I ; k)|$ $>\frac{1}{2} \cdot|I|^{2}$, and let $B$ be the set of $k$ 's for which $|B(K ; k)|>\frac{1}{2} \cdot|I|^{2}$.

Let $k^{\prime} \in B$. Then $k^{\prime} \geqq \inf B$, and $\left|B\left(I ; k^{\prime}\right)\right|>\frac{1}{2} \cdot|I|^{2}$. Thus it follows that $\left|A\left(I ; k^{\prime}\right)\right|<\frac{1}{2} \cdot|I|^{2}$, hence $k^{\prime} \notin A$. It is easily seen that if $k<\sup A$, then $k \in A$. Hence, $k^{\prime} \geqq \sup A$ from which it follows that $\inf B \geqq \sup A$.

Let us suppose that inf $B>\sup A$. Let inf $B>\xi>\sup A$. There are $(u, v)$ and $\left(u^{\prime}, v^{\prime}\right)$ in $I \times I$ such that $(g(u)-g(v)) /(u-v)>\xi$ and $\left(g\left(u^{\prime}\right)-g\left(v^{\prime}\right)\right) /\left(u^{\prime}-v^{\prime}\right)<\xi$. By the Mean Value Theorem for approximate derivatives (see [1]) there are $\zeta$ and $\zeta^{\prime}$ in $I$ such that

$$
\left(\frac{d g}{d x}\right)_{\mathrm{ap}}(\zeta)=\frac{g(u)-g(v)}{u-v} \text { and }\left(\frac{d g}{d x}\right)_{\mathrm{ap}}\left(\zeta^{\prime}\right)=\frac{g\left(u^{\prime}\right)-g\left(v^{\prime}\right)}{u^{\prime}-v^{\prime}} .
$$

By the Darboux property for approximate derivatives (see [2]), there is a $\sigma$ between $\zeta$ and $\zeta^{\prime}$ such that $(d g / d x)_{\text {ap }}(\sigma)=\xi$.

Hence, the set $M=\{(u, v) \in I \times I: \sup A<(g(u)-g(v)) /(u-v)$ $<\inf B\}$ is nonempty. In fact we will show the $M$ has positive measure. Let $\left(u_{0}, v_{0}\right) \in M$. Then the set

$$
M_{u_{0}}=\left\{v \in I: \sup A<\frac{\left(g\left(u_{0}\right)-g(v)\right)}{\left(u_{0}-v\right)}<\inf B\right\}
$$

has positive linear measure since $g$ is approximately continuous at $v_{0}$. For each $v^{\prime} \in M_{u_{0}}$, the set $\left\{u \in I: \sup A<\left(g(u)-g\left(v^{\prime}\right)\right) /(u-v)<\inf B\right\}$ has positive linear measure. Therefore the set $M$ has positive measure.

Since inf $B>\xi>\sup A,|B(I ; \xi)| \leqq \frac{1}{2} \cdot|I|^{2}$ and $|A(I ; \xi)| \leqq \frac{1}{2} \cdot|I|^{2}$. These inequalities imply that

$$
\left|\left\{(u, v) \in I \times I: \frac{g(u)-g(v)}{u-v} \geqq \xi\right\}\right| \geqq \frac{1}{2} \cdot|I|^{2}
$$

and

$$
\left|\left\{(u, v) \in I \times I: \frac{g(u)-g(v)}{u-v} \leqq \xi\right\}\right| \geqq \frac{1}{2} \cdot|I|^{2} .
$$

These inequalities are true for any $\xi$ between $\inf B$ and $\sup A$. Therefore 


$$
\left|\left\{(u, v) \in I \times I: \frac{g(u)-g(v)}{u-v} \geqq \inf B\right\}\right| \geqq \frac{1}{2} \cdot|I|^{2} .
$$

Similarly we obtain

$$
\left|\left\{(u, v) \in I \times I: \frac{g(u)-g(v)}{u-v} \leqq \sup A\right\}\right| \geqq \frac{1}{2} \cdot|I|^{2} .
$$

Hence it follows that

$$
\left|\left\{(u, v) \in I \times I: \sup A<\frac{g(u)-g(v)}{u-v}<\inf B\right\}\right|
$$

is equal to 0 , a contradiction. Therefore inf $B=\sup A$, and the proof is complete.

The interval function mentioned before the lemma is given by the equation in the statement of the lemma, that is, $\phi$ is defined on the collection of all subintervals $I$ of $J$ and the definition of $\phi(I)$ is as given above.

We also need the following lemma.

Lemma 3. Let $f$ be a measurable function of 2 real variables that is continuous with respect to the second variable and suppose that $I \times\left[y_{0}-1 / n, y_{0}+1 / n\right]$ is contained in the domain of $f$ for the real number $y_{0}$, the positive integer $n$, and the real interval I. If the set $S$ is defined by $S=\left\{(u, v) \in I \times I:\left(f\left(u, y_{0}\right)-f\left(v, y_{0}\right)\right) /(u-v)>a\right\}$ for some real number $a$, then the set $S_{n}$, defined by

$$
\begin{aligned}
& S_{n}=\left\{(u, v) \in S: \frac{f(u, y)-f(v, y)}{u-v} \geqq a\right. \\
& \left.\quad \text { for every } y \in\left(y_{0}-\frac{1}{n}, y_{0}+\frac{1}{n}\right)\right\},
\end{aligned}
$$

is a measurable set.

Proof. Let $\left\{\eta_{i}\right\}$ be a strictly decreasing sequence with $a$ as its limit. Let

$$
W_{i}(y)=\left\{(u, v) \in S: \frac{f(u, y)-f(v, y)}{u-v} \geqq \eta_{i}\right\} .
$$

For any given $i$ and $y, W_{i}(y)$ is measurable since

$$
\frac{f(u, y)-f(v, y)}{(u-v)}
$$


is a measurable function of $u$ and $v$ for a fixed $y$. We define $S_{n, i}$ $=\bigcap_{\boldsymbol{y} \in J(n)} W_{i}(y)$ where $J(n)$ denotes the interval $\left(y_{0}-1 / n, y_{0}+1 / n\right)$. Then

$$
\begin{aligned}
& S_{n, i}=\left\{(u, v) \in S: \frac{f(u, y)-f(v, y)}{(u-v)}\right. \geqq \eta_{i} \\
&\left.\quad \text { for every } y \in\left(y_{0}-\frac{1}{n}, y_{0}+\frac{1}{n}\right)\right\} .
\end{aligned}
$$

We will show that $S_{n, i}=\bigcap_{y \in Q(n)} W_{i}(y)$ where $Q(n)$ denotes all the rational numbers in $J(n)$. This will establish that $S_{n, i}$ is measurable for then $S_{n, i}$ will be the intersection of countably many measurable sets.

Let $\left(u_{0}, v_{0}\right) \in \bigcap_{y \in Q(n)} W_{i}(y)$, and let $y^{\prime}$ be any irrational in $\left(y_{0}-1 / n, y_{0}+1 / n\right)$. We need to verify that

$$
\frac{f\left(u_{0}, y^{\prime}\right)-f\left(v_{0}, y^{\prime}\right)}{y_{0}-v_{0}} \geqq \eta_{i} \text {. }
$$

Let $\left\{q_{m}\right\}$ be a sequence of rationals with $q_{m} \rightarrow y^{\prime}$. Then $\left(u_{0}, v_{0}\right)$ $\in W_{i}\left(q_{m}\right)$ for each $m$ for which $q_{m}$ is in $\left(y_{0}-1 / n, y_{0}+1 / n\right)$; thus $\left(f\left(u_{0}, q_{m}\right)-f\left(v_{0}, q_{m}\right)\right) /\left(u_{0}-v_{0}\right) \geqq \eta_{i}$ for $m$ sufficiently large. Since $f$ is continuous with respect to the second variable, it follows that $\left(f\left(u_{0}, y^{\prime}\right)-f\left(v_{0}, y^{\prime}\right)\right) /\left(u_{0}-v_{0}\right) \geqq \eta_{i}$. Therefore $\left(u_{0}, v_{0}\right)$ is in $W_{i}\left(y^{\prime}\right)$, and so $S_{n, i}=\bigcap_{y \in Q(n)} W_{i}(y)$ and $S_{n, i}$ is measurable. We observe that $S_{n}=\cup_{i=1}^{\infty} S_{n, i}$. Hence, each $S_{n}$ is measurable as was to be proved.

THEOREM 1. Let $G \subset R^{2}$ be a domain. If $f: G \rightarrow R$ is continuous relative to the second variable $(y)$ and the approximate partial derivative with respect to $x,(\partial f / \partial x)_{\mathrm{ap}}$, exists throughout $G$, then $(\partial f / \partial x)_{\mathrm{ap}}$ is in the first Baire class.

Proof. We will define a function $\Phi: G \times R^{+} \rightarrow R$ which is continuous with respect to $y$ for each fixed $x$ and $r$, and we will show that, for $\left(x_{0}, y_{0}\right) \in G, \lim \Phi(x, y, r)=(\partial f / \partial x)_{\text {ap }}\left(x_{0}, y_{0}\right)$ relative to $S\left(x_{0}, y_{0}\right)$ as $(x, y, r) \rightarrow\left(x_{0}, y_{0}, 0\right)$. The function $\Phi$ will be defined in terms of a convergent interval function which was defined by Casper Goffman and C. J. Neugebauer for the approximate derivative of a function of a real-variable (see [1]).

Let $\phi_{\boldsymbol{y}_{0}}$ denote the interval function of Goffman and Neugebauer defined on the linear sub-intervals of that part of the line $y=y_{0}$ which lies in $G$ (see below for the precise definition). We then let $\Phi\left(x_{0}, y_{0}, r_{0}\right)$ $=\phi_{y_{0}}\left(x_{0}-r_{0}, x_{0}, r_{0}\right)$ for $\left(x_{0}, y_{0}\right) G$ and $r_{0} \in R^{+}$. (Note: for a given $\left(x_{0}, y_{0}\right)$ it may happen that $\phi_{y_{0}}\left(x_{0}-r, x_{0}+r\right)$ is not defined for large 
values of $r$. We will ignore this since we are interested in the limit as $r \rightarrow 0$.)

Let $\left(x_{0}, y_{0}\right) \in G$ be fixed, and suppose $\left\{\left(x_{n}, y_{0}, r_{n}\right)\right\}$ is any sequence of points in $S\left(x_{0}, y_{0}\right)$ with $\left(x_{0}, y_{0}, 0\right)$ as limit. Since $\left|x_{n}-x_{0}\right| \leqq r_{n}$, the point $\left(x_{0}, y_{0}\right)$ is in each of the intervals $\left[x_{n}-r_{n}, x_{n}+r_{n}\right]$ on the line $y=y_{0}$. Therefore,

$$
\left(\frac{\partial f}{\partial x}\right)_{\mathrm{ap}}\left(x_{0}, y_{0}\right)=\lim _{n \rightarrow \infty} \phi_{\nu_{0}}\left(x_{n}-r_{n}, x_{n}+r_{n}\right)=\lim _{n \rightarrow \infty} \Phi\left(x_{n}, y_{0}, r_{n}\right) .
$$

We will now give the definition of $\Phi$ and show that it satisfies the "continuity" requirement of Lemma 1 . Suppose $\left(x_{0}, y_{0}, r_{0}\right)$ is a fixed point. Let $I$ denote the interval $\left[x_{0}-r_{0}, x_{0}+r_{0}\right]$ and define

$$
A(I ; y ; k)=\left\{(u, v) \in I \times I: \frac{f(u, y)-f(v, y)}{u-v}>k\right\} .
$$

Then the required interval function is defined by

$$
\phi_{\nu_{0}}(I)=\sup \left\{k: \frac{\left|A\left(I ; y_{0} ; k\right)\right|}{|I|^{2}}>\frac{1}{2}\right\} .
$$

Let $\eta=\phi_{y_{0}}(I)=\Phi\left(x_{0}, y_{0}, r_{0}\right)$ and let $\epsilon>0$ be given. The set

$$
S=\left\{(u, v) \in I \times I: \frac{f\left(u, y_{0}\right)-f\left(v, y_{0}\right)}{u-v}>\eta-\frac{\epsilon}{2}\right\}
$$

satisfies $|S|>\frac{1}{2} \cdot|I|^{2}$. Next define

$$
S_{n}=\left\{(u, v) \in S: \frac{f(u, y)-f(v, y)}{u-v}>\eta-\frac{\epsilon}{2}\right.
$$

$$
\text { for every } \left.y \in\left(y_{0}-\frac{1}{n}, y_{0}+\frac{1}{n}\right)\right\} \text {. }
$$

Since $f$ is continuous relative to $y$, each $(u, v) \in S$ is eventually in some $S_{n}$. Thus $S=\cup_{n=1}^{\infty} S_{n}$. Since the $S_{n}$ 's, are measurable (by Lemma 3 ), it follows that there is some $N$ such that $\left|\cup_{n=1}^{N} S_{n}\right|>\frac{1}{2} \cdot|I|^{2}$. Let $y^{\prime}$ be any number that satisfies $y_{0}-1 / n<y^{\prime}<y_{0}+1 / N$. We observe that

$$
\begin{aligned}
A\left(I ; y^{\prime} ; \eta-\epsilon\right) & =\left\{(u, v) \in I \times I: \frac{f\left(u, y^{\prime}\right)-f\left(v, y^{\prime}\right)}{u-v}>\eta-\epsilon\right\} \\
& \supset \bigcup_{n=1}^{N} S_{n} .
\end{aligned}
$$


Therefore $(|A(I ; y ; \eta-\epsilon)|) /|I|^{2}>\frac{1}{2}$, so that $\phi_{y^{\prime}}\left(x_{0}-r_{0}, \quad x_{0}+r_{0}\right)$ $\geqq \eta-\epsilon$.

Let

$$
B(I ; y ; k)=\left\{(u, v) \in I \times I: \frac{f(u, y)-f(v, y)}{u-v}<k\right\} .
$$

By Lemma 2, we have

$$
\sup \left\{k: \frac{\left|A\left(I ; y_{0} ; k\right)\right|}{|I|^{2}}>\frac{1}{2}\right\}=\inf \left\{k: \frac{\left|B\left(I ; y_{0} ; k\right)\right|}{|I|^{2}}>\frac{1}{2}\right\} \text {. }
$$

Let

$$
T=\left\{(u, v) \in I \times I: \frac{f\left(u, y_{0}\right)-f\left(v, y_{0}\right)}{u-v}<\eta+\frac{\epsilon}{2}\right\} .
$$

Then we have $|T|>\frac{1}{2} \cdot|I|^{2}$. Define

$$
T_{n}=\left\{(u, v) \in T: \frac{f(u, y)-f(v, y)}{u-v}<\eta+\frac{\epsilon}{2}\right.
$$

$$
\text { for every } \left.y \in\left(y_{0}-\frac{1}{n}, y_{0}+\frac{1}{n}\right)\right\} \text {. }
$$

Since $f$ is continuous relative to $y$, we have $T=\cup_{n=1}^{\infty} T_{n}$. The $T_{n}$ 's are measurable (the proof is similar to the proof of Lemma 3 ), hence there is an integer $K$ such that $\left|\cup_{n=1}^{K} T_{n}\right|>\frac{1}{2} \cdot|I|^{2}$. Let $y^{*}$ be any number that satisfies $y_{0}-1 / K<y^{*}<y_{0}+1 / K$. We observe that

$$
\begin{aligned}
B\left(I ; y^{*} ; \eta+\epsilon\right) & =\left\{(u, v) \in I \times I: \frac{f\left(u, y^{*}\right)-f\left(v, y^{*}\right)}{u-v}<\eta+\epsilon\right\} \\
& \supset \bigcup_{n-1}^{K} T_{n} .
\end{aligned}
$$

Therefore $\left|B\left(I ; y^{*} ; \eta+\epsilon\right)\right|>\frac{1}{2} \cdot|I|^{2}$ and so

$$
\phi_{y^{*}}\left(x_{0}-r_{0}, x_{0}+r_{0}\right) \leqq \eta+\epsilon .
$$

Let $M=\max \{N, K\}$. Then, if $y_{0}-1 / M<y<y_{0}+1 / M$, it follows that

$$
\begin{aligned}
& \left|\Phi\left(x_{0}, y, r_{0}\right)-\Phi\left(x_{0}, y_{0}, r_{0}\right)\right| \\
& \quad=\left|\phi_{y}\left(x_{0}-r_{0}, x_{0}+r_{0}\right)-\phi_{y_{0}}\left(x_{0}-r_{0}, x_{0}, r_{0}\right)\right|<\epsilon .
\end{aligned}
$$

Therefore $\Phi$ is continuous with respect to $y$ at $\left(x_{0}, y_{0}, r_{0}\right)$. Hence, by Lemma $1,(\partial f / \partial x)_{\text {ap }}$ is in the first Baire class.

REMARK. In addition to the theorem we previously mentioned, 
Tolstov showed that $\partial^{m} f / \partial x^{m}$ is in the first Baire class in a domain $G \subset R^{2}$ if $f$ is a linearly continuous function and if $\partial^{m} f / \partial x^{m}$ exists everywhere in $G$, and by means of an example, Tolstov showed that the partial derivative of a linearly continuous function of three variables need not be in the first Baire class. With the $n$-dimensional case of Lemma 1, it can be shown that if the function $f$ of three variables $(x, y, z)$ has "planar continuity" for each fixed value of $x$, then $\partial f / \partial x$ is in the first Baire class.

A linearly continuous function of two variables is in the first Baire class (e.g. see Kuratowski [3]), and the partial derivatives of such functions are in the first Baire class. Suppose $f: G \rightarrow R$ is linearly continuous and that $\partial^{m} f / \partial x^{m}$ exists everywhere in $G$. We observe that $\partial^{m} f / \partial x^{m}=(\partial / \partial x)\left(\partial^{m-1} f / \partial x^{m-1}\right)$, and that $\partial^{m-1} f / \partial x^{m-1}$ is in the first Baire class but is not necessarily linearly continuous. Thus we are led to ask if the hypothesis that $f: G \rightarrow R$ is in the first Baire class is sufficient in order that $\partial f / \partial x$ be in the first Baire class whenever $\partial f / \partial x$ exists throughout $G$. The following example gives a negative response to this question.

EXAmple. There is a function $f: R^{2} \rightarrow R$ which is in the first Baire class for which $\partial f / \partial x$ exists everywhere but is not in the first Baire class.

Let $\left\{r_{n}\right\}$ be a sequential ordering of the rational numbers in $R$. For each $n=1,2, \cdots$, let $g_{n}: R \rightarrow R$ be a differentiable function satisfying the conditions that $-1 / n \leqq g_{n}(x) \leqq 1 / n$ for all $x$ and $g_{n}^{\prime}(0)=1$. We define $f: R^{2} \rightarrow R$ as follows:

$$
f(x, y)= \begin{cases}0 & \text { if } y \text { is irrational } \\ g_{n}(x) & \text { if } y=r_{n} \text { for some } n .\end{cases}
$$

It is clear that $f$ is in the first Baire class and $\partial f / \partial x$ exists everywhere. However, on the $y$-axis $\partial f / \partial x$ is equal to zero at each point $(0, y)$ for which $y$ is irrational and $\partial f / \partial x$ is equal to one at each point $(0, y)$ for which $y$ is rational. Thus $\partial f / \partial x$ has no point of continuity on the perfect set which is the $y$-axis. Therefore $\partial f / \partial x$ is not in the first Baire class.

\section{BIBLIOGRAPHY}

1. C. Goffman and C. J. Neugebauer, On approximate derivatives, Proc. Amer. Math. Soc. 11 (1960), 962-966.

2. F. Hausdorff, Set theory, Chelsea, New York, Translation of 3rd edition (1937) of Mengenlehre.

3. C. Kuratowski, Topologie, I, 3 ème éd., Monogr. Mat. 21 (1952), 285.

4. G. P. Tolstov, On partial derivatives, Amer. Math. Soc. Transl. No. 69, (1952). 